\title{
Application of Cubature Information Filter for Underwater Target Path Estimation
}

\section{Guduru Naga Divya* and Sanagapallea Koteswara Rao \\ Department of Electronics and Communication Engineering, Koneru Lakshmaiah Education Foundation, Vaddeswaram, Guntur, AP, India}

\begin{abstract}
Bearings-only tracking plays a pivotal role in passive underwater surveillance. Using noisy sonar bearing measurements, the target motion parameters (TMP) are extensively estimated using the extended Kalman filter (EKF) because of its simplicity and low computational load. The EKF utilizes the first order approximation of the nonlinear system in estimation of the TMP that degrades the accuracy of estimation due to the elimination of the higher order terms. In this paper, the cubature Kalman filter (CKF) that captures the system nonlinearity upto third order is proposed to estimate the TMP. The CKF is further extended using the information filter (IF) to provide decentralized data fusion, hence the filter is termed as cubature information filter (CIF). The results are generated using Matlab with Gaussian assumption of noise in measurements. Monte-Carlo simulation is done and the results demonstrate that the CIF accuracy is same as that of UKF and this indicates the usefulness of the algorithm for state estimation in underwater with the required accuracy.

Keywords: Bearings-only tracking, cubature Kalman filter, information filter, state estimation, stochastic signal processing
\end{abstract}

\section{INTRODUCTION}

Surveillance is the most significant feature of maritime warfare. The observer submarine is in waters doing their surveillance job. The observer moves at low speeds such that it

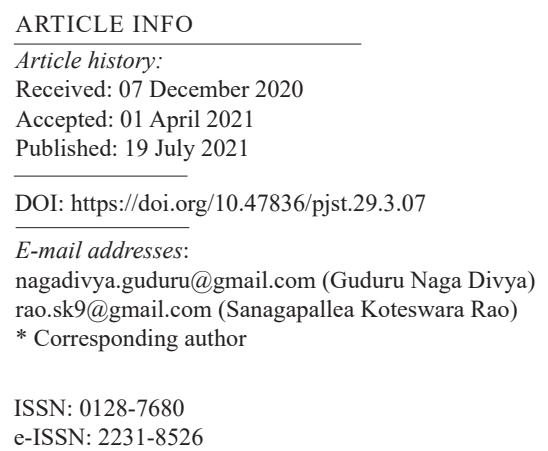
does not radiate much noise during tracking of the target. Passive target tracking is the calculation of target's trajectory merely from measurements of signals emerging from the target. These signals could be machinery noise from a target and its detection is usually indicated by an increase in energy above the ambient at certain bearing. Passive 
mode helps the observer from not being tracked by the target ship. Observer equipped with hull mounted sonar can pick up the radiated noise of target and can generate bearing measurement of the target. This process is called 'bearings-only target tracking'(BOT).

In BOT process, only one type of measurement namely bearing measurements are available and there are two components of range that is in $\mathrm{x}$ and $\mathrm{y}$ directions, speed and course to be estimated. Hence the process becomes unobservable for most scenarios. So, observer has to carry out maneuver, for observability of the process and to estimate target motion parameters (TMP) (Koteswararao, 2018). Often observer has to carry out number of S-maneuvers to obtain the estimated TMP with the desired accuracy, whenever measurements are corrupted with high noise.

Tracking process in underwater environment contains dynamic perturbations like time to time change in the environment, velocity of the vehicle, self-noise of the observer and so on. These disturbances or noises that are internal or external to the observer affect the performance of hull mounted array of the sonar and other transducers generating the target and observer measurements. In other words, the target bearing generated is inaccurate and corrupted with noise. The observer contains gyro and log generate observer course and speed respectively. These measurements are also contaminated with noise. However, smoothing of these course and speed measurements is trivial and so this is not covered in this research manuscript.

From the available noisy information, obtaining the information of target regarding its velocity and range with respect to the observer is called target motion analysis (TMA) and in passive listening mode is most popularly called 'Bearings-only target tracking (BOT)'. This is an active research since decades and researchers are trying to a) improve the accuracy in estimated solution using various signal processing algorithms b) generate less complex, easy to implement, less convergence time procedures and so on.

The classical estimator with respect to nonlinear processes is extended Kalman filter (EKF). However, EKF is unreliable for BOT, as in-depth knowledge regarding plant noise and Jacobian of measurement dynamic process are not sufficient. Recently proposed unscented Kalman filter (UKF) (Wan \& Van Der Merwe, 2000) and cubature Kalman filter (CKF) (Arasaratnam \& Haykin, 2009; Ding \& Balaji, 2012) are Jacobian-free filters available to nullify the disadvantages of the EKF.

$\mathrm{CKF}$ is the recently developed nonlinear filtering algorithm based on spherical radial cubature rule. CKF has strong mathematical basis in the selection of cubature points and numerical stability than UKF. The computational load using CKF is relatively lower as it uses only $2 * L_{1}\left(L_{1}\right.$ is the dimension of the system) cubature points and UKF uses $2 * L_{1}+1$ sigma points to propagate the state and covariance. When the dimension of the nonlinear system is greater than 3 , there is a chance that the chosen sigma points may be negative which render the negative covariance matrix definite whereas cubature points in CKF are 
positive indicating the positive definiteness of the covariance matrix. Hence CKF algorithm is considered for this BOT application. Its extension, cubature information filter (CIF) (Pakki et al., 2011; Arasaratnam \& Chandra, 2015; Jiang \& Cai, 2018) is used such that it is suitable for nonlinear process and at the same time for the implementation of data available from multiple sensors. (In future if measurements are available from passive sonar, periscope, and active radar/sonar, CIF is very much useful for multi sensor data fusion configuration.)

Kalman filter processes all the sensors measurements' centrally to obtain the solution thereby incurring high computational load on the digital computer. Therefore, the information filter is proposed, as it can be extended straight to design multi-sensor fusion algorithms and the computations are also simple than the conventional Kalman filter. Also the initialization of information filter (IF) is simple as it is independent of the system priori state. While determining the inverse of a high dimension augmented matrix, the digital computer may fail which is a big drawback of centralized fusion. The data fusion algorithms can be implemented efficiently in a decentralized way using IF. In comparison to the traditional centralized data fusion algorithms, the decentralized structure is more powerful and efficient in terms of computation and communication (Mutambara, 1998).

In information filter (IF), information state vector and information matrix play an important role. The inverse of the covariance matrix is named as information matrix (IM). The information state vector (ISV) is the product of the IM and the target state vector. In EKF, the target state vector and its covariance matrix are propagated in time domain recursive processing. In IF, the concept of EKF's recursive propagation is applied to IM and ISV which is termed as extended information filter (EIF). EIF is combined with CKF algorithm, named as cubature information filter (CIF) is applied to BOT and the same can be applied in future for multi-sensor applications. The details about CIF are given next section.

Mathematical modeling, design and implementation in Matlab of CIF are carried out as per the requirements of BOT. Initialization of state vector and its covariance matrix are chosen such that the algorithm works for all scenarios. Simulator is developed to feed various tactical scenarios. Simulator also generate the true TMP and observer position. Performance of the algorithm is evaluated against several scenarios in Monte-Carlo simulation. Acceptance criterion is chosen based on some particular weapon guidance and accordingly the convergence of the solution is calculated. The results for one typical scenario is presented.

The aim of this research work is to estimate TMP like range, course, speed and smoothed bearing as early as possible in sea environment. Once target path is estimated, the weapon can be released on to the target. The measurements are assumed to be available from observer's hull mounted sonar. The block diagram of TMP is shown in Figure 1. Further, TMP is used to calculate weapon pre-set parameters for releasing weapon on to a target. 


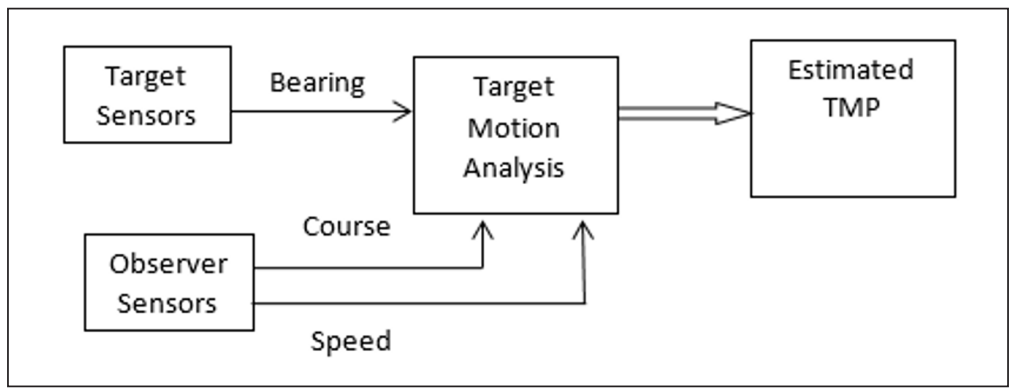

Figure 1. Block diagram of target motion parameters

\section{MATHEMATICAL MODELLING}

\section{Target State Equation}

In BOT, the target-observer scenario is modelled mathematically based on following assumptions. Initially, the observer is located at $(0,0)$ and the target is located at position $\mathrm{P}$ at some range as shown in Figure 2.

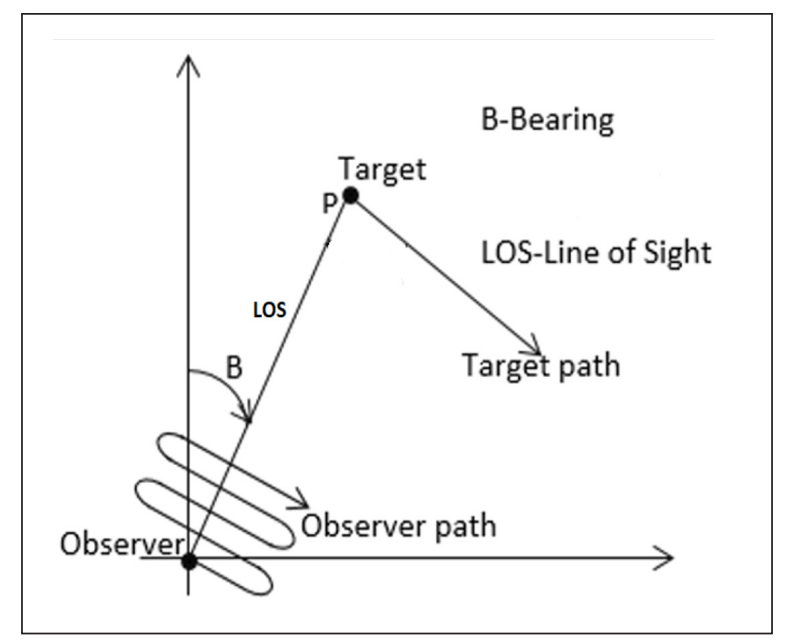

Figure 2. Target and Observer movements

The observer and target are assumed to be heading with constant course and speed. The target's relative state vector $(X s)$ in Cartesian coordinates w.r.t. observer at input sample $\left(\Gamma_{s t}\right)$ is represented as Equation 1.

$$
X_{s}\left(\Gamma_{s t}\right)=\left[\begin{array}{llll}
\dot{r}_{x}\left(\Gamma_{s t}\right) & \dot{r}_{y}\left(\Gamma_{s t}\right) & r_{x}\left(\Gamma_{s t}\right) & r_{y}\left(\Gamma_{s t}\right)
\end{array}\right]^{T}
$$

Where $\dot{r}_{x}\left(\Gamma_{s t}\right), \dot{r}_{y}\left(\Gamma_{s t}\right), r_{x}\left(\Gamma_{s t}\right), r_{y}\left(\Gamma_{s t}\right)$ are the components of speed and range in $\mathrm{x}$ and $\mathrm{y}$ coordinates respectively at input sample number $\Gamma_{s t}$. The subsequent instant relative state vector based on the current instant $\left(X_{s}\left(\Gamma_{s t}+1, \Gamma_{s t}\right)\right)$ is given by Equation 2. 


$$
X_{s}\left(\Gamma_{s t}+1, \Gamma_{s t}\right)=A\left(\Gamma_{s t}+1, \Gamma_{s t}\right) X_{s}\left(\Gamma_{s t}\right)+N_{g} \varepsilon\left(\Gamma_{s t}\right)[
$$

Where $A\left(\Gamma_{s t}+1, \Gamma_{s t}\right)$ is matrix representing the system dynamics given as Equation 3.

$$
A\left(\Gamma_{s t}+1, \Gamma_{s t}\right)=\left[\begin{array}{cccc}
1 & 0 & 0 & 0 \\
0 & 1 & 0 & 0 \\
t & 0 & 1 & 0 \\
0 & t & 0 & 1
\end{array}\right]
$$

Where ' $t$ ' represents the interval at which samples are obtained. $\varepsilon\left(\Gamma_{s t}\right)$ is the system noise that is assumed to obey Gaussian distribution having mean $=0$ and covariance $Q$. The system noise gain matrix $\left(N_{g}\right)$ is given in matrix form as Equation 4.

$$
N_{g}=\left[\begin{array}{cc}
t & 0 \\
0 & t \\
t^{2} / 2 & 0 \\
0 & t^{2} / 2
\end{array}\right]
$$

The system noise covariance $(Q)$ is given in matrix form as Equation 5.

$$
\mathrm{Q}\left(\Gamma_{s t}\right)=\sigma^{2}\left[\begin{array}{cccc}
t^{2} & 0 & t^{3} / 2 & 0 \\
0 & t^{2} & 0 & t^{3} / 2 \\
t^{3} / 2 & 0 & t^{4} / 4 & 0 \\
0 & t^{3} / 2 & 0 & t^{4} / 4
\end{array}\right]
$$

Where $\sigma^{2}$ is system noise variance.

\section{Measurement Equation}

The measured bearing angle $b_{m}$ is given by Equation 6 .

$$
\mathrm{b}_{m}\left(\Gamma_{s t}+1\right)=\tan ^{-1}\left(\frac{r_{x}\left(\Gamma_{s t}+1\right)}{r_{y}\left(\Gamma_{s t}+1\right)}\right)
$$

The measurement model equation $(Z)$ at sample $\Gamma_{s t}+1$ is specified by Equation 7 .

$$
Z\left(\Gamma_{s t}+1\right)=H\left(\Gamma_{s t}+1\right) X_{s}\left(\Gamma_{s t}+1\right)+\gamma_{\mathrm{b}}
$$

Where $\mathrm{H}$ is the matrix representation of the relationship between $X_{S}$ and $Z$. The $\gamma_{\mathrm{b}}$ is the noise of the measurement. Here the assumption is that the measurement noise follows Gaussian/uniform distribution, with variance $\sigma_{\mathrm{b}}^{2}$.

The measurement vector $Z\left(\Gamma_{s t}\right)$ is given by Equation 8 .

$$
Z\left(\Gamma_{s t}\right)=b_{m}\left(\Gamma_{s t}\right)
$$


This tracking problem, the aim is to estimate the state vector $X_{S}\left(\Gamma_{s t}\right)$, from a set of measurements $Z\left(\Gamma_{s t}\right)=\left[z(1) z(2) \ldots . z\left(\Gamma_{s t}\right)\right]^{T}$.

\section{Cubature Kalman Filter Algorithm}

For many practical applications, the use of EKF is not the best option, as it works well only in a 'mild' nonlinear environment and can therefore degrade efficiency. The CKF is the nearest known Bayesian approximation to the nonlinear system with the Gaussian assumption. CKF doesn't require Jacobian evaluation making it attractive for state estimation. The steps in CKF are organized in two sections i.e. prediction and updation as given below.

\section{Prediction}

1. The sigma points are calculated using Equation 9.

$$
X_{s}\left(i, \Gamma_{s t}\right)=m\left(\Gamma_{s t}\right)+\sqrt{P\left(\Gamma_{s t}\right)} \chi(i) \quad \mathrm{i}=1, \ldots \ldots .2 L_{1}
$$

Where $m\left(\Gamma_{s t}\right)$ and $P\left(\Gamma_{s t}\right)$ is the mean and covariance at input sample number $\Gamma_{s t}$ and $L_{1}$ is the dimension of the system. The unit sigma points $\chi(i)$ are defined as Equation 10.

$$
\chi(i)=\left\{\begin{aligned}
\sqrt{L_{1}} e_{i}, i & =1, \ldots ., 2 L_{1} \\
-\sqrt{L_{1}} e_{i-L_{1}}, i & =n+1, \ldots ., 2 L_{1}
\end{aligned}\right.
$$

where $e_{i}$ denotes a unit vector in the direction of the coordinate axis $i$.

2. The sigma points are propagated through the dynamic model as in Equation 11.

$$
\hat{X}_{s}\left(i, \Gamma_{s t}+1\right)=A\left(X_{s}\left(i, \Gamma_{s t}+1\right)\right), \quad \mathrm{i}=1, \ldots ., 2 L_{1}
$$

3. The predicted mean $m\left(\Gamma_{s t}+1, \Gamma_{s t}\right)$ and the predicted covariance $P\left(\Gamma_{s t}+1, \Gamma_{s t}\right)$ are calculated using Equations 12 and 13.

$$
\begin{aligned}
m\left(\Gamma_{s t}+1, \Gamma_{s t}\right)= & \frac{1}{2 L_{1}} \sum_{i=1}^{2 L_{1}} \hat{X}_{s}\left(i, \Gamma_{s t}+1\right) \\
P\left(\Gamma_{s t}+1, \Gamma_{s t}\right)= & \frac{1}{2 L_{1}} \sum_{i=1}^{2 L_{1}}\left(\hat{X}_{s}\left(i, \Gamma_{s t}+1\right)-m\left(\Gamma_{s t}+1, \Gamma_{s t}\right)\right)\left(\hat{X}_{s}\left(i, \Gamma_{s t}+1\right)-\right. \\
& \left.m\left(\Gamma_{s t}+1, \Gamma_{s t}\right)\right)^{T}+Q\left(\Gamma_{s t}\right)
\end{aligned}
$$




\section{Updation}

1. The sigma points are formed as Equation 14.

$X_{s}\left(i, \Gamma_{s t}+1, \Gamma_{s t}\right)=m\left(\Gamma_{s t}+1, \Gamma_{s t}\right)+\sqrt{P\left(\Gamma_{s t}+1, \Gamma_{s t}\right)} \chi(i), \quad \mathrm{i}=1, \ldots . ., 2 L_{1}$

Where the unit sigma points are defined as in Equation 9.

2. Sigma points are propagated through the measurements model as Equation 15.

$$
\hat{Z}\left(i, \Gamma_{s t}+1\right)=H\left(X_{s}\left(i, \Gamma_{s t}+1, \Gamma_{s t}\right)\right), \quad \mathrm{i}=1 \ldots 2 L_{1}
$$

3. The predicted mean $\mu\left(\Gamma_{s t}+1\right)$, the predicted covariance of the measurement $P_{X X}\left(\Gamma_{s t}+1\right)$, and the cross-covariance of the state and the measurement $P_{X Z}\left(\Gamma_{s t}+1\right)$ are calculated using Equation 16-18.

$$
\begin{aligned}
& \mu\left(\Gamma_{s t}+1\right)=\frac{1}{2 L_{1}} \sum_{i=1}^{2 L_{1}} \hat{Y}\left(i, \Gamma_{s t}+1\right) \\
& P_{X X}\left(\Gamma_{s t}+1\right)=\frac{1}{2 L_{1}} \sum_{i=1}^{2 L_{1}}\left(\hat{Z}\left(i, \Gamma_{s t}+1\right)-\mu\left(\Gamma_{s t}+1\right)\right)\left(\hat{Z}\left(i, \Gamma_{s t}+1\right)-\mu\left(\Gamma_{s t}+1\right)\right)^{T}+Q\left(\Gamma_{s t}+1\right)
\end{aligned}
$$

$$
P_{X Z}\left(\Gamma_{s t}+1\right)=\frac{1}{2 L_{1}} \sum_{i=1}^{2 L_{1}}\left(X\left(i, \Gamma_{s t}+1, \Gamma_{s t}\right)-m\left(\Gamma_{s t}+1, \Gamma_{s t}\right)\right)\left(\hat{Z}\left(i, \Gamma_{s t}+1\right)-\mu\left(\Gamma_{s t}+1\right)\right)^{T}
$$

4. The filter gain $K\left(\Gamma_{s t}+1\right)$ and the filtered state mean $m\left(\Gamma_{s t}+1\right)$ and covariance $P\left(\Gamma_{s t}+1\right.$ are calculated using Equations 19-21.

$$
\begin{aligned}
& K\left(\Gamma_{s t}+1\right)=P_{X X}\left(\Gamma_{s t}+1\right) P_{X Z}^{-1}\left(\Gamma_{s t}+1\right) \\
& m\left(\Gamma_{s t}+1\right)=m\left(\Gamma_{s t}+1, \Gamma_{s t}\right)+K\left(\Gamma_{s t}+1\right)\left[Z\left(\Gamma_{s t}+1\right)-\mu\left(\Gamma_{s t}+1\right)\right] \\
& P\left(\Gamma_{s t}+1\right)=P\left(\Gamma_{s t}+1, \Gamma_{s t}\right)-K\left(\Gamma_{s t}+1\right) P_{X Z}\left(\Gamma_{s t}+1\right) K^{T}\left(\Gamma_{s t}+1\right)
\end{aligned}
$$

\section{Information Filter}

The mathematical modeling of the information filter is given as follows and the detailed explanation for the filter is given in (Pakki et al., 2011).

The information state vector is represented by $I X s$, at time index $\Gamma_{s t}$ is given by Equation 22.

$$
I X_{s}\left(\Gamma_{s t}\right)=I M\left(\Gamma_{s t}+1, \Gamma_{s t}\right) * X_{s}\left(\Gamma_{s t}+1, \Gamma_{s t}\right)
$$


Where $I M$ is given as Equation 23 .

$$
I M\left(\Gamma_{s t}+1, \Gamma_{s t}\right)=\left(P\left(\Gamma_{s t}+1, \Gamma_{s t}\right)\right)^{-1}
$$

By substituting the covariance from EIF (Pakki et al., 2011), Equation 22 is given as Equation 24.

$$
\operatorname{IM}\left(\Gamma_{s t}+1, \Gamma_{s t}\right)=\left[A\left(\Gamma_{s t}\right)\left(I M\left(\Gamma_{s t}+1, \Gamma_{s t}+1\right)\right)^{-1} A\left(\Gamma_{s t}\right)+Q\left(\Gamma_{s t}\right)\right]^{-1}
$$

where $P\left(\Gamma_{s t}+1, \Gamma_{s t}\right)$ is predicted covariance matrix

$Q\left(\Gamma_{s t}\right)$ is covariance of plant noise

$A\left(\Gamma_{s t}\right)$ is transition matrix

The updated information state vector, $I X_{s}\left(\Gamma_{s t}+1, \Gamma_{s t}+1\right)$, and the updated information matrix, $I M\left(\Gamma_{s t}+1, \Gamma_{s t}+1\right)$, are given by Equations 25 and 26 .

$$
\begin{aligned}
& I X_{s}\left(\Gamma_{s t}+1, \Gamma_{s t}+1\right)=I X_{s}\left(\Gamma_{s t}+1, \Gamma_{s t}\right)+i\left(\Gamma_{s t}\right) \\
& I M\left(\Gamma_{s t}+1, \Gamma_{s t}+1\right)=I M\left(\Gamma_{s t}+1, \Gamma_{s t}\right)+I\left(\Gamma_{s t}\right)
\end{aligned}
$$

Where (Equations 27 and 28)

$$
\begin{aligned}
i\left(\Gamma_{s t}\right)= & I M\left(\Gamma_{s t}+1, \Gamma_{s t}\right) P_{x y}\left(\Gamma_{s t}+1, \Gamma_{s t}\right) Q^{-1}\left(\Gamma_{s t}\right)\left[v\left(\Gamma_{s t}\right)+\right. \\
& P_{x y}{ }^{T}\left(\Gamma_{s t}+1, \Gamma_{s t}\right) I M^{T}\left(\Gamma_{s t}+1, \Gamma_{s t}\right) I X_{s}\left(\Gamma_{s t}+1, \Gamma_{s t}\right) \\
I\left(\Gamma_{s t}\right)= & I M\left(\Gamma_{s t}+1, \Gamma_{s t}\right) P_{x y}\left(\Gamma_{s t}+1, \Gamma_{s t}\right) Q^{-1}\left(\Gamma_{s t}\right) P_{x y}{ }^{T}\left(\Gamma_{s t}+1, \Gamma_{s t}\right) I M\left(\Gamma_{s t}+1, \Gamma_{s t}\right)
\end{aligned}
$$

Where the measurement residual, $v\left(\Gamma_{s t}\right)$, is given by Equation 29 .

$$
v\left(\Gamma_{s t}\right)=Z\left(\Gamma_{s t}\right)-\hat{Z}\left(\Gamma_{s t}\right)
$$

\section{Cubature Information Filter}

The updated state vector and the updated information matrix obtained from Equations 25 and 26 are used to update the state and covariance obtained from Equations 16-18. Hence the state and covariance using CIF are obtained as follows.

The updated state vector and covariance matrix can are given by Equations 30 and 31 .

$$
\begin{aligned}
& X_{s}\left(\Gamma_{s t}+1, \Gamma_{s t}\right)=I M\left(\Gamma_{s t}+1, \Gamma_{s t}+1\right) \backslash I X_{s}\left(\Gamma_{s t}+1, \Gamma_{s t}+1\right) \\
& P\left(\Gamma_{s t}+1, \Gamma_{s t}+1\right)=I M\left(\Gamma_{s t}+1, \Gamma_{s t}+1\right)
\end{aligned}
$$


The performance of the CIF is compared with the standard filter unscented Kalman Filter (UKF). For the detailed mathematical modeling of UKF refer (Wan \& Van Der Merwe, 2000).

\section{RESULTS AND DISCUSSION}

In target tracking process, there appear two types of perturbations. The first type is selfnoise of the observer and the environment. The second type is the difference between the process understood in mathematical modelling of the target's state and the real system dynamics. The source of error for the second type appears due to half knowledge about system nonlinearities and order of the models, plant noise variations due to environment and so on. For example, general assumption is that the target moves at constant velocity, with the disturbances in velocity considered as white noise having very small variance. It may not be true always in all circumstances, due to change in sea environment.

In general, in any modern sonar system, sophisticated hardware with state of art signal processing is used to reduce self-noise. During normal sea state conditions, number of field trials will be carried out to find out self-noise and environmental noise. The same will be used as the input measurement covariance matrix. All raw bearing measurements are corrupted by additive zero-mean Gaussian noise. Here bearing measurements are considered with respect to Y-axis, $0^{\circ}-360^{\circ}$ and clockwise positive. The measurement interval is 1 s i.e., at every second bearing measurement is available and the number of measurement samples taken for simulation is 800 samples. As the measurements are available at every second, the simulation time is taken as $800 \mathrm{~s}$. Also Monte- Carlo simulation is carried out to give the confidence of the CIF algorithm. The performance of the CIF algorithm with bearing measurements is evaluated for several geometries. Acceptance criteria gives the acceptable level of errors in estimated values. Only bearing measurement gives the information about the target and this bearing is corrupted with noise due to underwater environment. Hence the errors in the estimated parameters are only reduced using the filtering algorithms but cannot be removed $100 \%$. So there is necessity to know how much error can be accepted in the estimate. This acceptable level of errors in estimates is called acceptance criterion. The acceptable level of errors in the estimated parameters is $3 \sigma$ for single run and $1 \sigma$ for 100 Monte-Carlo runs. Based on particular weapon guidance algorithm the acceptable errors are chosen in an estimated range, course and speed as less than or equal to $10 \%$ of true range, $5^{\circ}$ and $1 \mathrm{~m} / \mathrm{s}$ respectively for single run $(3 \sigma)$ and $3.33 \%$ of true range, $1.67^{\circ}$ and $0.33 \mathrm{~m} / \mathrm{s}$ respectively for 100 Monte-Carlo runs $(1 \sigma)$.

\section{Initialization of State Vector}

The following assumptions are made to estimate the target state vector initially as follows. There is no knowledge about the range measurement, the velocity components are initialized 
as $5 \mathrm{~m} / \mathrm{s}$. Based on the Sonar range of the day the initial range is taken as $5000 \mathrm{~m}$. Hence the initial target state vector $X_{S}(0,0)$ is taken as Equation 32 .

$$
X_{s}(0,0)=\left[\begin{array}{lll}
5 & 5 & 5000 \sin B_{m}(0) \quad 5000 \cos B_{m}(0)
\end{array}\right]
$$

\section{Initialization of Covariance Matrix}

Assuming the initial state vector follows uniform density function, the initial covariance matrix $P(0,0)$ which is a diagonal matrix. These elements are given by Equation 33 .

$$
P(0,0)=\operatorname{Diag}\left(4 X_{s}^{2}(i) / 12\right) \quad \text { where } i=1,2,3,4
$$

\section{Performance Evaluation of the Algorithm}

The measurement is assumed to be available at each second. The turning rate of the observer in underwater is $0.5 \mathrm{deg} / \mathrm{s}$. The target is moving at constant velocity. The simulation study is done using Matlab on a personal computer. For making the process observable and thereafter to obtain TMA (i.e., range, course and speed of the target), in general, the observer carries out S-maneuver preferably on the line of sight in azimuth plane. Change in vehicle's course or speed or both is called maneuver. Here course maneuver is implemented as speed maneuver is not recommended due to tactics limitations. In general, one maneuver by observer attains observability of the process and is sufficient to generate solution with required accuracy, in normal sea state conditions. The algorithm is evaluated against numbers of scenarios. The scenarios chosen for the algorithm evaluation are tabulated Table 1. In Table 1, the scenarios are chosen in such a way to suit the real time environment. The initial range is chosen based on the Sonar range of the day. The detectable range of passive sonar vary from few meters to $10 \mathrm{~km}$ depending on many parameters like ambient (background) noise and self-noise which is termed as Sonar range of the day. Also the homing range of the sophisticated torpedo is $3000 \mathrm{~m}$. So, keeping in mind these points the initial range of the target is moderately assumed to be around $4500 \mathrm{~m}$. The observer velocity is chosen so that there is less self-noise. Generally, a submarine is used for surveillance in underwater. The submarine can go at a maximum speed of 20knots (18 $\mathrm{m} / \mathrm{s}$ ). However, this will increase the self-noise very much. Tracking the target in passive mode is considered so that the observer is safe from being tracked by the target. There is necessity that the observer should move at lower speeds to reduce the self-noise. So the observer speed is initialized as $5 \mathrm{~m} / \mathrm{s}$. This also helps to get the observability of the target. The target course is taken such that the target is heading towards the bow of the observer. Initial bearing is taken such that the target is always heading towards bow of the observer and also for simplicity purpose all the initial bearing angles are taken such that they fall in 
first quadrant. The standard deviation (S.D) of noise in the bearing measurement is taken as $0.17^{\circ}$ i.e. the maximum error in the measurement is $0.5^{\circ}$.

Table 1

Scenarios chosen for evaluation

\begin{tabular}{ccccccc}
\hline S. no. & $\begin{array}{c}\text { Target range } \\
(\mathrm{m})\end{array}$ & $\begin{array}{c}\text { Observer } \\
\text { speed }(\mathrm{m} / \mathrm{s})\end{array}$ & $\begin{array}{c}\text { Target speed } \\
(\mathrm{m} / \mathrm{s})\end{array}$ & $\begin{array}{c}\text { Target course } \\
(\mathrm{deg})\end{array}$ & $\begin{array}{c}\text { Target bearing } \\
(\mathrm{deg})\end{array}$ & $\begin{array}{c}\text { S.D of noise in } \\
\text { bearing }(\mathrm{deg})\end{array}$ \\
\hline 1 & 4500 & 9 & 11 & 165 & 20 & 0.17 \\
2 & 5000 & 7 & 10 & 160 & 20 & 0.17 \\
3 & 5000 & 7 & 10 & 170 & 20 & 0.17 \\
4 & 4000 & 8 & 12 & 135 & 30 & 0.17 \\
5 & 4500 & 9 & 11 & 160 & 40 & 0.17 \\
6 & 5000 & 7 & 10 & 145 & 20 & 0.17 \\
\hline
\end{tabular}

Note. S.D- standard deviation

For detailed discussion, let us consider scenario 5 in Table 1. A target ship is moving at $11 \mathrm{~m} / \mathrm{s}$ at a course of $160^{\circ}$, making an initial bearing angle of $40^{\circ}$ with the observer. The initial range between the target and observer is $4500 \mathrm{~m}$. The observer moves at a speed of $9 \mathrm{~m} / \mathrm{s}$. The bearing measurements are corrupted with white Gaussian noise whose standard deviations (S.D) are $0.17^{\circ}$. As per the acceptance criteria, the convergence times for single run and Monte-Carlo simulation using CIF and UKF for the scenarios of Table 1 are tabulated in Tables 2 and 3, respectively.

As per the acceptance criteria considered for single run, estimated range is said to be converged when the error in the range estimate is less than or equal to $10 \%$ of true range and never diverges thereafter in the period of simulation and the same procedure is followed for course and speed. So in the period of 800 s simulation time using CIF, the range estimate error is less than or equal to $10 \%$ of true range at $247 \mathrm{~s}$ first time and converged thereafter without any divergence. Similarly, the course estimate error is less than or equal to $5^{\circ}$ at $327 \mathrm{~s}$ first time and converged thereafter without any divergence and speed estimate error is less than or equal to $1 \mathrm{~m} / \mathrm{s}$ at $304 \mathrm{~s}$ first time and converged thereafter without any divergence. It means that the total solution is said to be converged at 327 seconds. Similarly using UKF, the range estimate error is less than or equal to $10 \%$ of true range at $196 \mathrm{~s}$ first time and converged thereafter without any divergence. Similarly, the course estimate error is less than or equal to $5^{\circ}$ at $328 \mathrm{~s}$ first time and converged thereafter without any divergence and speed estimate error is less than or equal to $1 \mathrm{~m} / \mathrm{s}$ at $307 \mathrm{~s}$ first time and converged thereafter without any divergence. It means that the total solution is said to be converged at 328 seconds.

As per the acceptance criteria considered for single run, the estimated range is said to be converged when the error in the range estimate is less than or equal to $3.33 \%$ of true range and never diverges thereafter in the period of simulation and the same procedure 
is followed for course and speed. So in the period of 800 s simulation time using CIF, the range estimate error is less than or equal to $3.33 \%$ of true range at $267 \mathrm{~s}$ first time and converged thereafter without any divergence. Similarly, the course estimate error is less than or equal to $1.67^{\circ}$ at $351 \mathrm{~s}$ first time and converged thereafter without any divergence and speed estimate error is less than or equal to $0.33 \mathrm{~m} / \mathrm{s}$ at $344 \mathrm{~s}$ first time and converged thereafter without any divergence. It means that the total solution is said to be converged at 351 seconds. Similarly, using UKF, the range estimate error is less than or equal to $3.33 \%$ of true range at $261 \mathrm{~s}$ first time and converged thereafter without any divergence. Similarly, the course estimate error is less than or equal to $1.67^{\circ}$ at $369 \mathrm{~s}$ first time and converged thereafter without any divergence and speed estimate error is less than or equal to 0.33 $\mathrm{m} / \mathrm{s}$ at $363 \mathrm{~s}$ first time and converged thereafter without any divergence. It means that the total solution is said to be converged at 369 seconds.

Scenarios 1,4 and 5 converged faster using CIF (consider total convergence times from Monte-Carlo simulation) at 333, 375 and 351 seconds respectively whereas using UKF the same scenarios converged at 354, 439 and 369 seconds respectively. Similarly,

Table 2

Convergence time in seconds of the chosen scenarios for single run

\begin{tabular}{|c|c|c|c|c|c|c|c|c|}
\hline \multirow[t]{3}{*}{ S.no } & \multicolumn{8}{|c|}{ Convergence time in seconds for single run } \\
\hline & \multicolumn{4}{|c|}{$\mathrm{CIF}$} & \multicolumn{4}{|c|}{ UKF } \\
\hline & $\mathrm{R}$ & $\mathrm{S}$ & $\mathrm{C}$ & $\mathrm{CT}$ & $\mathrm{R}$ & $\mathrm{S}$ & $\mathrm{C}$ & CT \\
\hline 1 & 326 & 308 & 262 & 326 & 341 & 266 & 264 & 341 \\
\hline 2 & 302 & 351 & 352 & 352 & 217 & 227 & 226 & 227 \\
\hline 3 & 340 & 361 & 355 & 361 & 278 & 235 & 159 & 278 \\
\hline 4 & 198 & 149 & 216 & 216 & 198 & 149 & 216 & 216 \\
\hline 5 & 247 & 304 & 327 & 327 & 196 & 307 & 328 & 328 \\
\hline 6 & 236 & 353 & 370 & 370 & 191 & 176 & 226 & 226 \\
\hline
\end{tabular}

Note. R, Range; S, Speed; C, Course; CT, Convergence Time

Table 3

Convergence time in seconds of the chosen scenarios for 100 runs

\begin{tabular}{|c|c|c|c|c|c|c|c|c|}
\hline \multirow[t]{3}{*}{ S.no } & \multicolumn{8}{|c|}{ Convergence time in seconds for 100 runs } \\
\hline & \multicolumn{4}{|c|}{ CIF } & \multicolumn{4}{|c|}{ UKF } \\
\hline & $\mathrm{R}$ & $\mathrm{S}$ & $\mathrm{C}$ & $\mathrm{CT}$ & $\mathrm{R}$ & $\mathrm{S}$ & $\mathrm{C}$ & $\mathrm{CT}$ \\
\hline 1 & 333 & 331 & 327 & 333 & 354 & 314 & 334 & 354 \\
\hline 2 & 324 & 375 & 372 & 375 & 256 & 315 & 278 & 315 \\
\hline 3 & 358 & 384 & 375 & 384 & 294 & 311 & 200 & 311 \\
\hline 4 & 306 & 349 & 374 & 374 & 407 & 431 & 439 & 439 \\
\hline 5 & 267 & 344 & 351 & 351 & 261 & 363 & 369 & 369 \\
\hline 6 & 352 & 400 & 404 & 404 & 225 & 213 & 284 & 284 \\
\hline
\end{tabular}

Note. R, Range; S, Speed; C, Course; CT, Convergence Time 
scenarios 2,3 and 6 converged faster using UKF (consider total convergence times from Monte-Carlo simulation) at 315, 311 and 284 seconds respectively whereas using CIF the same scenarios converged at 375,384 and 404 seconds respectively. For scenarios 1,4 and 5, CIF is better while UKF is better 2,3 and 6 scenarios. From Tables 2 and 3, it is emphasized that the CIF and UKF are giving total convergence time for all the scenarios comparably. The true and estimated paths of target and observer using CIF and UKF are shown in Figure 3 and 4, respectively. The estimated path of the target in Figure 3 is so

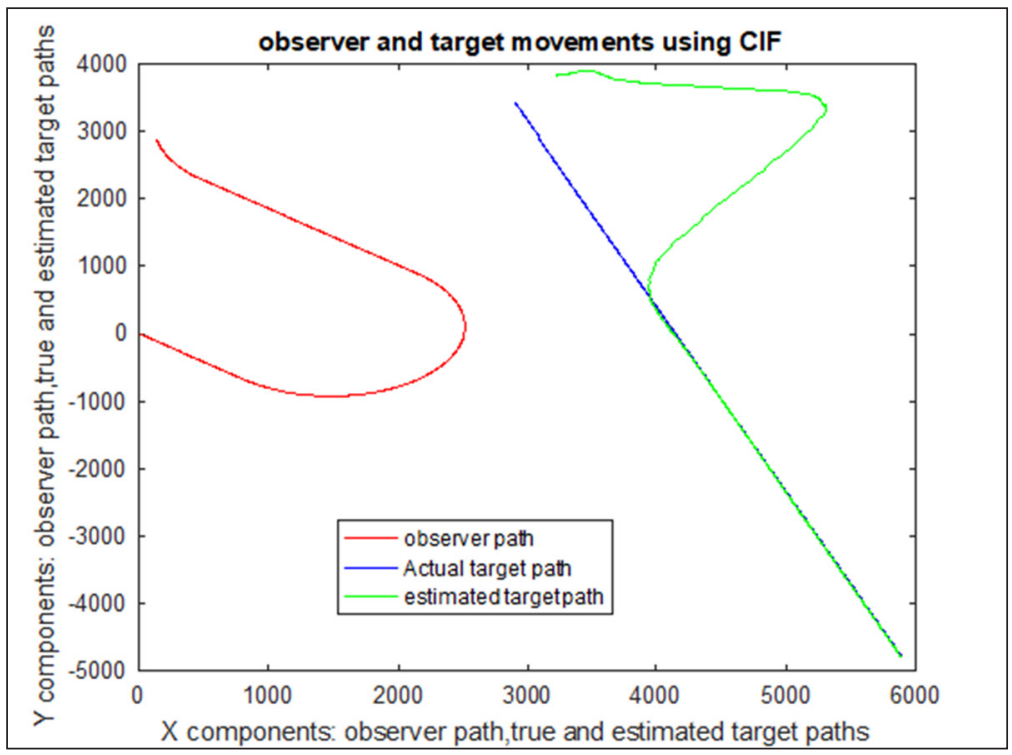

Figure 3. Observer and target movements using CIF

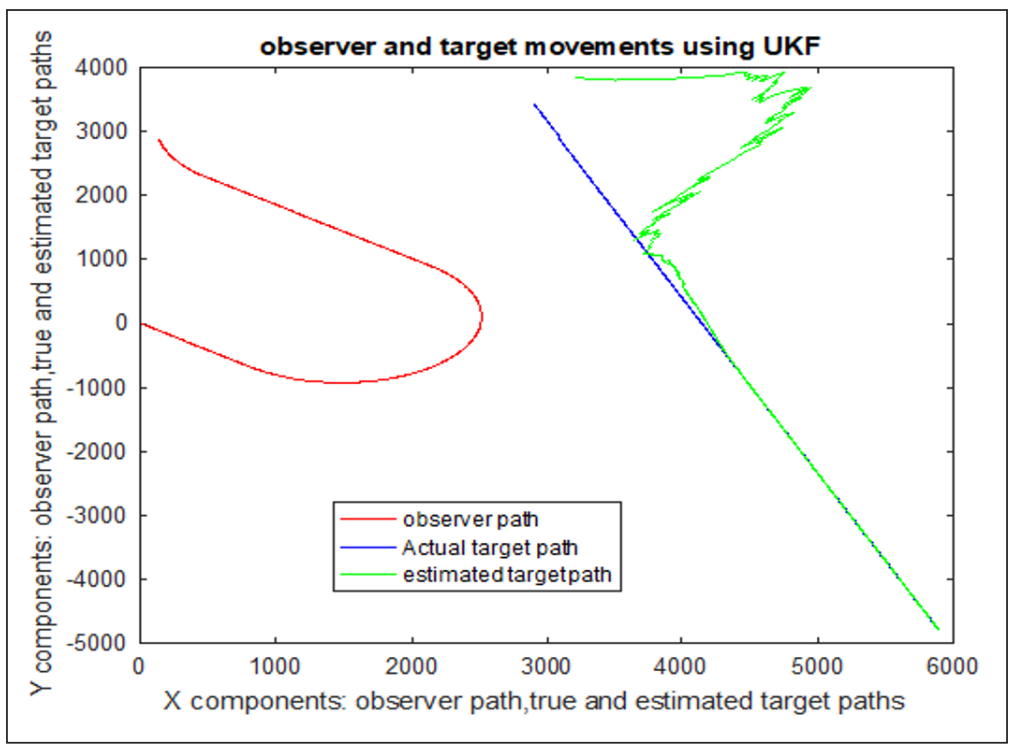

Figure 4. Observer and target movements using UKF 
smooth indicating CIF stability. The estimated path using UKF is zigzag indicating the filter instability when compared to CIF.

The errors in estimated range, speed and course using CIF and UKF are shown in Figures 5a to 5c, respectively. Similarly, the RMS errors in estimated range, speed and course using CIF and UKF are shown in Figure 6a to 6c, respectively.

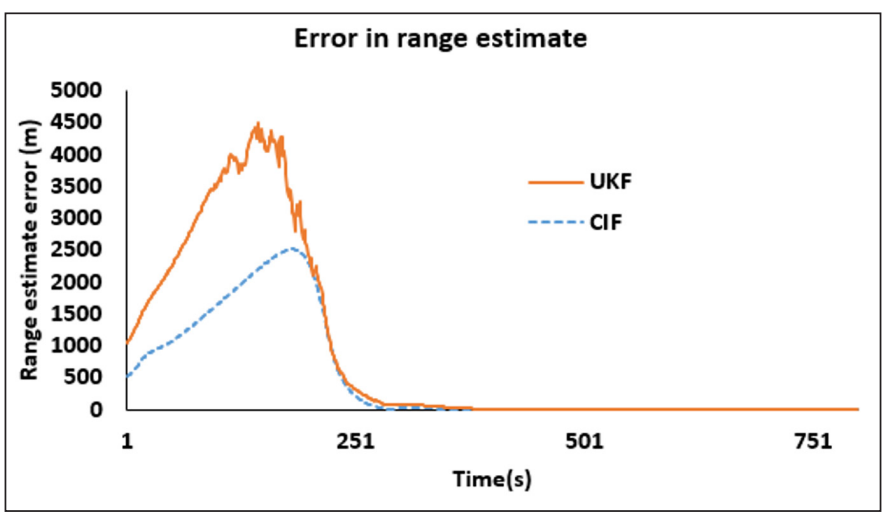

(a)

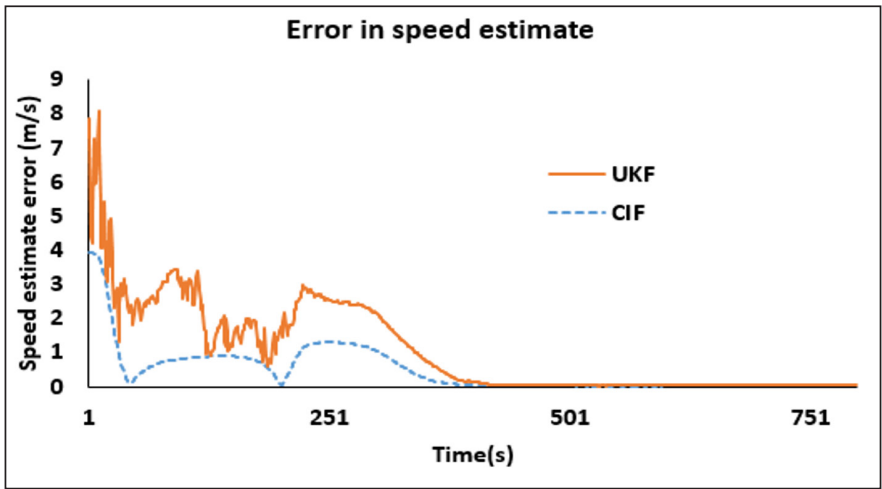

(b)

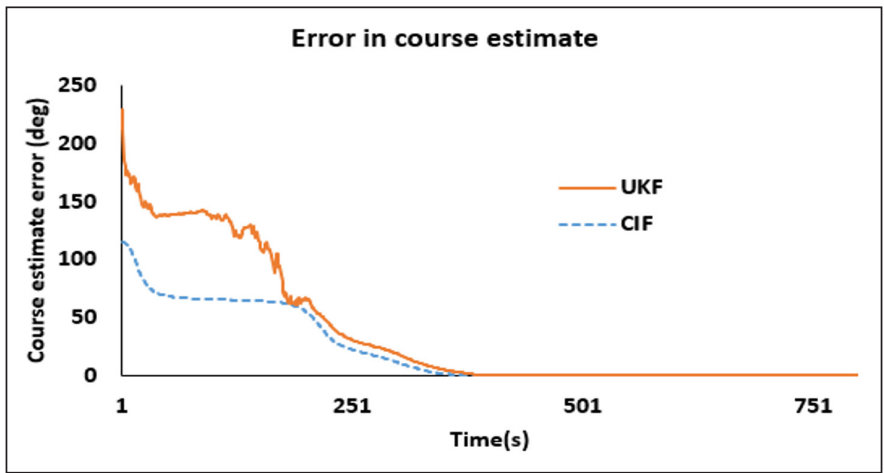

(c)

Figure 5. Error for Scenario 5: (a)Range estimate; (b) Speed estimate; and (c) Course estimate 
From the Figure 6a, it is understood that the RMS error in range estimate using the CIF (blue dashed line) is very much less which indicates the convergence nature of CIF. Also the curve is very smooth which indicates the CIF filter stability. The RMS error in range estimate of target using the CIF and UKF almost converged at the same time i.e., $267 \mathrm{~s}$ and 261s (Table 3), respectively. These all indicate the usefulness of the algorithm for

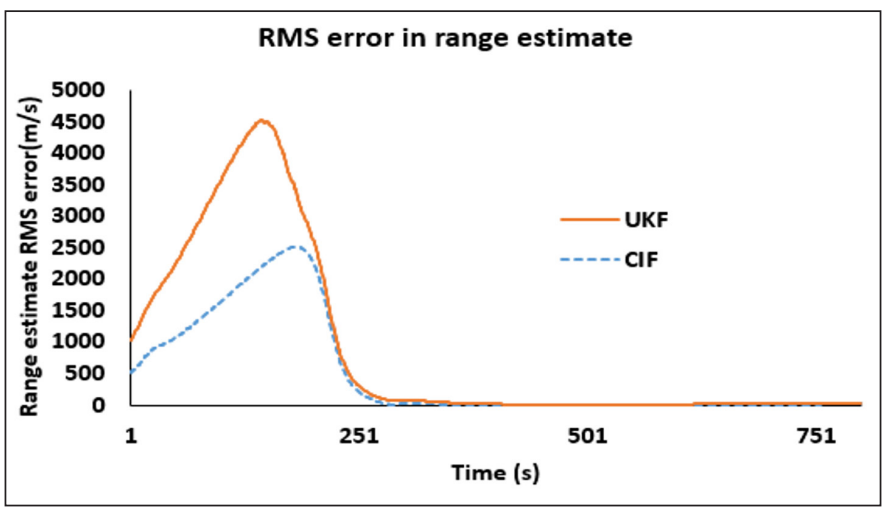

(a)

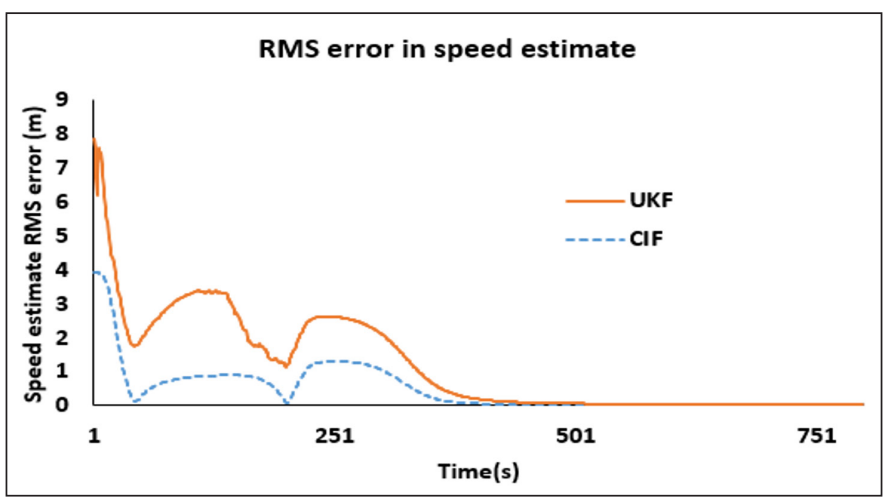

(b)

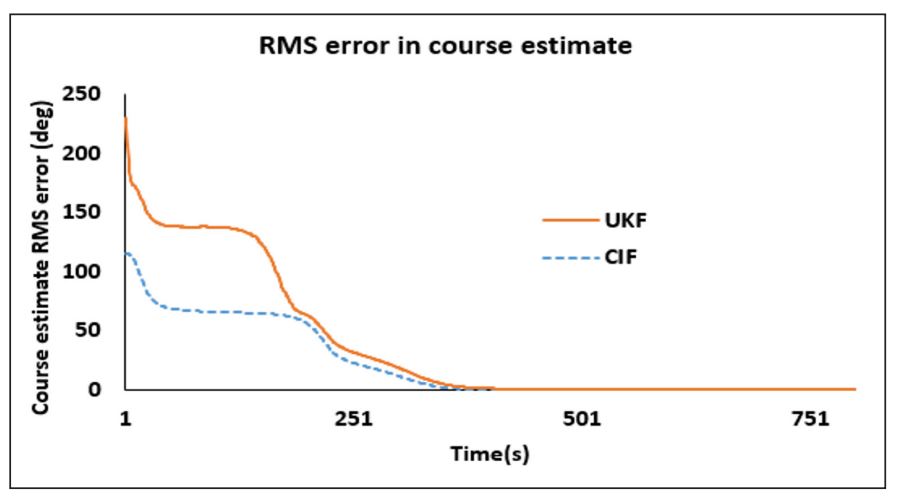

(c)

Figure 6. RMS error for Scenario 5: (a) Range estimate; (b) Speed estimate; and (c) Course estimate 
state estimation in underwater with the required accuracy. The same can be observed from Figure 6b (Speed estimate RMS error for Scenario 5) and Figure 6c (Course estimate RMS error for Scenario 5) for RMS error in speed and course estimates. As the computations are straightforward in CIF, the RMS error value is found to be less when compared to UKF enabling the CIF to withstand more unstable and higher order system nonlinearity and measurement noises. From the literature till date, UKF is found to be giving solution accurately for systems with Taylor's series of third order. From the analysis done in this research, CIF is found to be giving solution similar to UKF.

\section{CONCLUSION}

Target tracking is proposed in underwater using CIF, a less complex and easy to implement algorithm. One of the best advantages of CIF over other filters is the decentralized data fusion ability as the corrected ISV and IM can be obtained by simply adding the associated values to the updated ISV and IMs. Simulator is developed to feed various scenarios and evaluate the algorithm. Performance evaluation of CIF algorithm is carried out in simulation mode and the results are presented for typical scenarios. The convergence times using UKF and CIF are almost near for all the scenarios except for difference of $120 \mathrm{~s}$ in scenario 6. CIF is found to be appealing simulation results for BOT using single sensor in comparison with UKF with the help of straightforward computations. Keeping in view, the smaller number of computations and decentralized data fusion ability of CIF, CIF is very much useful for state estimation in underwater.

\section{ACKNOWLEDGEMENT}

The authors are grateful to the authorities of Koneru Lakshmaiah Education Foundation (Deemed to be university), Vaddeswaram, Guntur, Andhra Pradesh, India for their encouragement towards the research and publication of the paper.

\section{REFERENCES}

Arasaratnam, I., \& Chandra, K. B. (2015). Cubature information filters: Theory and applications to multisensor fusion. In Multisensor Data Fusion (pp. 216-229). CRC Press.

Arasaratnam, I., \& Haykin, S. (2009). Cubature kalman filters. IEEE Transactions on Automatic Control, 54(6), 1254-1269. https://doi.org/10.1109/TAC.2009.2019800

Ding, Z., \& Balaji, B. (2012, October 22-25). Comparison of the unscented and cubature Kalman filters for radar tracking applications. In IET International Conference on Radar Systems (Radar 2012). Glasgow, UK. https://doi.org/10.1049/cp.2012.1695

Jiang, H., \& Cai, Y. (2018). Adaptive fifth-degree cubature information filter for multi-sensor bearings-only tracking. Sensors, 18(10), Article 3241. https://doi.org/10.3390/s18103241 
Koteswararao, S. (2018). Bearings-only tracking: Observer maneuver recommendation. IETE Journal of Research, 67(2), 193-204. https://doi.org/10.1080/03772063.2018.1535917

Mutambara, A. G. O. (1998). Decentralized estimation and control for multisensor systems. CRC Press.

Pakki, K., Chandra, B., Gu, D. W., \& Postlethwaite, I. (2011). Cubature information filter and its applications. In Proceedings of the 2011 American Control Conference (pp. 3609-3614). IEEE Conference Publication. https://doi.org/10.1109/ACC.2011.5990913

Wan, E. A., \& Van Der Merwe, R. (2000, October). The unscented Kalman filter for nonlinear estimation. In Proceedings of the IEEE 2000 Adaptive Systems for Signal Processing, Communications, and Control Symposium (Cat. No. 00EX373) (pp. 153-158). IEEE Conference Publication. https://doi.org/10.1109/ ASSPCC.2000.882463 
